\title{
Global aerodynamic instability of twin cylinders in cross flow
}

\author{
Md. Mahbub Alam ${ }^{1, *}$ and J.P. Meyer ${ }^{2}$ \\ ${ }^{1}$ Department of Mechanical and Automation Engineering \\ Shenzhen Graduate School, Harbin Institute of Technology \\ Shenzhen, China \\ ${ }^{2}$ Department of Mechanical and Aeronautical Engineering \\ University of Pretoria, Pretoria 0002, South Africa \\ *Email: alamm28@yahoo.com, alam@hitsz.edu.cn
}

\begin{abstract}
This paper comprises an in-depth physical discussion of the flow-induced vibration of two circular cylinders in view of the time-mean lift force on stationary cylinders and interaction mechanisms. The gap-spacing ratio $T / D$ is varied from 0.1 to 5 and the attack angle $\alpha$ from $0^{\circ}$ to $180^{\circ}$ where $T$ is the gap width between the cylinders and $D$ is the diameter of a cylinder. Mechanisms of interaction between two cylinders are discussed based on time-mean lift, fluctuating lift, flow structures and flow-induced responses. The whole regime is classified into seven interaction regimes, i.e., no interaction regime; boundary layer and cylinder interaction regime; shear-layer/wake and cylinder interaction regime; shear-layer and shear-layer interaction regime; vortex and cylinder interaction regime; vortex and shear-layer interaction regime; and vortex and vortex interaction regime. Though a single non-interfering circular cylinder does not correspond to a galloping following quasi-steady galloping theory, two circular cylinders experience violent galloping vibration due to shear-layer/wake and cylinder interaction as well as boundary layer and cylinder interaction. A larger magnitude of fluctuating lift communicates to a larger amplitude vortex excitation.
\end{abstract}

Keywords: cylinders, forces, flow structures, instabilities, interactions.

\section{Introduction}

While much is known of the flow physics around a single isolated cylinder, not much is known of the fluid dynamics around a cylinder neighbored by another. There is no doubt that flow physics around two cylinders is much more complex and complicated than that around a single cylinder, because of 
interference between the cylinders (Alam et al. 2011). Mutual flow interaction between two structures makes the wake either very excited or tranquil depending on the spacing between the structures. The excited wake-enhancing forces may in some cases cause a catastrophic failure of the structures. The study of the aerodynamics of two closely separated structures is thus of both fundamental and practical significance. Vibration problems are frequently encountered for structures consisting of multiple cylinders such as electric power lines, flow sensor tubing, etc. The resulting vibrations depend strongly on cylinder configuration (relative to flow), pitch spacing, cylinder diameters and flow conditions. Cross-flow-induced vibration is the most important problem in various fields, and is known to have caused many failures in various industrial components.

The instability of slender structures has received the attention of many scientists during decades, its theoretical foundations being well established and understood in Parkinson and Smith (1964), Novak $(1969,1972)$ and Simiu and Scanlan (1996). Besides theoretical work, great effort has been devoted to experimental study of the instability features of many bodies having different cross sections. One of the reasons for such studies is the fact that buildings and other slender structural elements are built more and more frequently using new techniques that involve weight-saving materials (thus reducing the overall stiffness) and innovative cross-sectional geometries. In consequence, when designing certain structures such as particularly high and slender buildings, one may find that critical velocities of aeroelastic instabilities such as vortex-induced excitation and galloping are within the design wind speed.

Zdravkovich (1987) divided the possible arrangements of two cylinders into four regions: (i) the proximity interference region, where the flow around one cylinder affects the other; (ii) the wake interference region, where the near-wake flow of the upstream cylinder is unaffected by the downstream one; but the downstream one is significantly affected by the upstream cylinder; (iii) the proximity and wake interference region, where both proximity and wake interference are significant; and (iv) the no-interference region, where the wake of one cylinder does not affect the other. Sumner et al. (2000) conducted flow visualization and particle image velocimetry (PIV) measurements for $T / D=1.0 \sim 5.0, \alpha=$ 
$0^{\circ} \sim 90^{\circ}$ and Reynolds number $\operatorname{Re}=850-1900$ (see Fig. 1 for the definitions of the symbols), and divided the $T / D-\alpha$ plane into three: (1) the single-body flow regime, $T / D=1.0 \sim 1.125$ and $\alpha=0^{\circ} \sim 90^{\circ}$, where two cylinders act like an isolated body with a single vortex-shedding frequency; (2) the small incidence angle regime, $T / D>1.125$ and $\alpha=0^{\circ} \sim 20^{\circ}$, where shear layer reattachment or the impingement of vortices onto the downstream cylinder takes places; and (3) the large incidence angle regime, $T / D>1.125$, $\alpha=20^{\circ} \sim 90^{\circ}$, where vortex pairing, splitting, enveloping and synchronizing occur. Price and Paidoussis (1984), Zdravkovich and Pridden (1977), as well as Gu and Sun (1999) measured time-mean drag and lift forces acting on two staggered cylinders, placing most of the emphasis is on the downstream cylinder. A review of flow around two cylinders was made by Sumner (2010).

Practically no structure is perfectly rigid, hence it is worthy to gain physical insight into the flow-induced response of the structure. Bokaian and Geoola (1984a) investigated the case of two identical cylinders in tandem and staggered arrangements where the downstream one was fixed and the upstream one both-end-spring-mounted, allowing both ends to vibrate at the same amplitude (two-dimensional model) in the cross-flow direction only. They reported galloping vibration generated at a spacing ratio of $T / D<0.8\left(\alpha=25^{\circ}\right), T / D \leq 0.75\left(\alpha=0^{\circ}\right)$ and vortex excitation $(\mathrm{VE})$ at other $T / D$ and $\alpha$. Bokaian and Geoola (1984b) also investigated the other case where the upstream cylinder was fixed and the downstream one was free to oscillate. Depending on $T / D$, the cylinder exhibited either only galloping $(T / D$ $\left.=0.59, \alpha=0^{\circ}\right)$ or only VE $\left(T / D>1.5, \alpha=0^{\circ}\right)$ or a combined VE and galloping $\left(T / D>0.5, \alpha=0^{\circ}\right)$, or a separated VE and galloping $(1.0 \leq T / D \leq 1.5)$. Note that the vibration always occurs at the natural frequency $f_{n}$ of the cylinder. The VE corresponded to vibration occurring near the reduced velocity $U_{r}(=$ $U_{\infty} / f_{n} / D, U_{\infty}$ is the free-stream velocity) where the natural vortex-shedding frequency $f_{v}$ is close to $f_{n}$. On the other hand, the galloping vibrations persist for higher $U_{r}$ corresponding to a higher $f_{v}$ than $f_{n}$. In Bokaian and Geoola (1984a, b), the investigated ranges of $T / D, \alpha$ and mass-damping factor $m * \zeta$ were 0.09 $\sim 4,0^{\circ} \sim 70^{\circ}$ and $0.018 \sim 0.2$, respectively, where $m^{*}$ is the mass ratio and $\zeta$ is the damping ratio.

Brika and Laneville (1999) performed an experimental investigation of the dynamic response of a long 
flexible circular cylinder in the wake of a stationary geometrically similar cylinder for $T / D=7.5 \sim 25.5$ and $U_{r}=4 \sim 25\left(\operatorname{Re}=5 \times 10^{3} \sim 2.7 \times 10^{4}\right)$. The system had a very low $m^{*} \zeta$ of 0.00007 . They observed that, as $T / D$ increases from 7.5 to 25.5 , the value of $U_{r}$ at maximum vibration amplitude in the vortex excitation regime is slightly reduced and the regime covers a smaller range of $U_{r}$. Compared with an isolated cylinder, the vortex excitation occurs at higher reduced velocities and the VE region is wider; this region was twice as large for $T / D=10.5$ and decreases with increased spacing. For $T / D=7.5 \sim 9$, the cylinder exhibited a combination of vortex-induced and wake-galloping oscillations. Hover and Triantafyllou (2001) examined the response of and forces on the spring-mounted downstream cylinder for $T / D=4.75$. They observed both vortex-resonance and galloping to occur when $U_{r}$ was varied from 2 to 17 , with changing $\mathrm{f}_{\mathrm{n}}$ and constant $U_{\infty}$ corresponding to $\operatorname{Re}=3.05 \times 10^{4}$. Brika and Laneville (1997) investigated the effect on the downstream cylinder response of the upstream cylinder, stationary or vibrating for $T / D=7 \sim 25$. When the upstream cylinder is stationary, the response of the downstream cylinder was no longer hysteretic and it was strongly influenced by the spacing between the cylinders. The VE regime decreased with increasing $T / D$ and appears on a wider range of the reduced velocity. Laneville and Brika (1999) coupled two identical cylinders $\left(T / D=7 \sim 25, m^{*} \zeta=0.00007\right)$ mechanically by thin wires, allowing them to vibrate in in-phase and out-of-phase mode. They found that the response of the cylinder is more complex and dependent on the coupling mode. Huera-Huarte and Bearman (2011) conducted experiments on flow-induced responses of two tandem cylinders for $L / D=1$ to 3 at $m^{*} \zeta=0.043$. The upstream cylinder experienced larger vibrations than the rear one for small gap distances at small $U_{r}$, when the shedding frequency was close to its natural frequency. The downstream cylinder exhibited galloping with large amplitudes at high $U_{r}$ for the largest gap separations.

Kim et al. $(2008,2009)$ and Alam and Kim (2009) conducted a systematic investigation on flow-induced response characteristics of two circular cylinders $\alpha=0^{\circ}, 5^{\circ}, 10^{\circ}, 15^{\circ}, 25^{\circ}, 45^{\circ}, 60^{\circ}$, and $90^{\circ}, T / D$ ranging from 0.1 to 3.2. At each position $(\alpha, T / D)$ of the cylinders, dependence of vibration-amplitude-to-diameter ratio $a / D$ on reduced velocity $U_{r}$ was examined. 
The objectives of this study were to (i) classify possible interaction mechanisms for two stationary rigid cylinders, and (ii) correlate interaction mechanisms, lift forces and flow-induced responses of the cylinders mounted elastically. The possible range of $\alpha=0^{\circ} \sim 180^{\circ}$ was considered with $T / D=0.1 \sim 5.0$. Flow-induced response results are incorporated from literature published by the current authors and others.

\section{Experimental details}

Fluid force measurements were conducted in Fluid Mechanics Laboratory of Kitami Institute of Technology, Japan, inside a closed-circuit wind tunnel with a $2.2-\mathrm{m}$-long test section of $0.3 \mathrm{~m}$ in width and $1.2 \mathrm{~m}$ in height. Two circular cylinders of a diameter $D=49 \mathrm{~mm}$, made of brass, spanned horizontally across the test section width. The free-stream velocity, $U_{\infty}$, was $17 \mathrm{~m} / \mathrm{s}$, resulting in $\operatorname{Re}\left(\equiv U_{\infty} D / v\right)=5.5 \times$ $10^{4}$, where $v$ is the kinematic viscosity of air. The flow non-uniformity was within $\pm 0.2 \%$ (rms) inside the central cross-sectional area of $0.24 \mathrm{~m} \times 0.95 \mathrm{~m}$ in the test section, and the longitudinal turbulence intensity was less than $0.5 \%$ in the absence of the cylinders. More details of the tunnel can be found in Alam et al. (2005). A schematic diagram of the cylinder arrangement appears in Fig. 1, along with the definitions of symbols. The Cartesian coordinate system was defined such that the origin was at the center of Cylinder $A$, with the $x$ - and y-axis along the streamwise and lateral directions, respectively.

Fluid forces were measured over a small spanwise length of the cylinders using load cells. The cylinder to be measured was built in with an active ('live') section of a spanwise $0.92 D$ length and two dummy sections. The active section, placed between the two dummy sections, corresponded to the mid-span of the cylinder and was installed with a load cell that consisted of four semiconductor strain gages. One of the dummy sections was also instrumented with another load cell of the same configuration. The load cell inside the active section measured a combination of fluid forces and forces due to vibration transmitted from outside through the cylinder support, while that inside the dummy section measured the latter forces only. Hence, the fluid forces acting on the active section could be calculated by subtracting the output of the load cell inside the dummy section from that of the load cell inside the active section.

Measurements were done for $\alpha=0^{\circ}, 10^{\circ}, 25^{\circ}, 45^{\circ}, 60^{\circ}, 75^{\circ}, 90^{\circ}, 105^{\circ}, 120^{\circ}, 135^{\circ}, 155^{\circ}$, 
$170^{\circ}$, and $180^{\circ}$, for the spacing ratio of $T / D=0.1 \sim 5$. Tuning of $T / D$ was $T / D=0.1,0.2,0.3,0.5,0.6,0.7$, $0.8,0.9,1.1,1.2,1.5,1.8,2.1,2.4,2.7,3.0,3.5,4.0,4.5$ and 5.0.

Flow visualization was carried out in a water channel with a $250 \times 350 \mathrm{~mm}$ working section and $1.5 \mathrm{~m}$ in length. Two circular tubes with identical diameters of $20 \mathrm{~mm}$ were used. The Reynolds number in the water channel experiment was 350 . The flow was visualized by using the hydrogen bubble technique, involving a platinum wire of $0.02 \mathrm{~mm}$ in diameter.

\section{Lift forces}

Time-averaged lift coefficient $\left(C_{L}\right)$ and fluctuating $(\mathrm{rms})$ lift coefficient $\left(C_{L f}\right)$ are measured of the traversing cylinder (cylinder $B$ ) for the whole ranges of $\alpha$ and $T / D$ mentioned in section 2. Contours of measured $C_{L}$ and $C_{L f}$ in a $T / D-\alpha$ plane are presented in Fig. 2. In the scale bars, the color or the range marked by black '*' indicates the value of a single isolated cylinder. The result can be described with reference to Fig. 1 in which Cylinder $A$ is fixed, and traversing of Cylinder $B$ is done with variation of the two parameters $T / D$ and $\alpha$, which suffice to determine the possible arrangement of the two cylinders. It may be noted that Cylinder $B$ acts as the downstream cylinder for $|\alpha|<90^{\circ}$ and the upstream cylinders for $|\alpha|>90^{\circ}$, i.e. the left and right sides of a contour map show the values of coefficient of the upstream and downstream cylinders, respectively. At the peripheries of the middle and outer circles, the values of $T / D$ are 0.0 and 5.0, respectively. Upward (+ve y-direction) $C_{L}$ is considered as positive.

The $C_{L}$ in the downstream region (right half) is highly sensitive to $T / D$ and $\alpha$; however, that in the upstream region (left half) retains single-cylinder values except for $|\alpha|=135^{\circ}-180^{\circ}, T / D<0.4-1.0$, and $|\alpha|=90^{\circ}-135^{\circ}, T / D<1.3-0.4$. The $C_{L}$ around the cylinder for $T / D<0.5$ varies greatly with change in $\alpha$ from $0^{\circ}$ to $360^{\circ}$. The minimum (most negative) values of $C_{L}=-1.03$ and $-1.15 \sim-1.25$ occur at $\alpha=155^{\circ}$, $T / D=0.3$ and $\alpha=10^{\circ}, T / D=0.8 \sim 1.1$, respectively. At the respective conjugate positions, $C_{L}$ increases to a maximum. On the other hand, $C_{L f}$ is extremely small for smaller spacing, i.e., $T / D<2-3$ depending on $\alpha$ (Fig. 2(b)) and remarkably high for $\alpha=-35^{\circ}$ to $35^{\circ}, T / D>2.5-3.0$. Hence the interference between the cylinders not only has a negative effect with increasing forces, but also a positive effect with reducing 
forces on the cylinder. Its effect, however, depends on $\alpha$ and $T / D$. It is expected that at different values of $T / D$ and $\alpha$, interaction mechanisms between the cylinders will be different, hence $C_{L}$ and $C_{L f}$ are strong functions of $T / D$ and $\alpha$.

\section{Interaction Mechanisms}

Though a cylinder is called a wake generator, it normally generates not only a wake but also boundary layers, shear layers, and vortices. When one cylinder is neighbored by another, the two cylinders may be connected or interacted by boundary layers, shear layer, vortex and wake. Therefore it is possible that a cylinder may experience complex interaction mechanisms where cylinder, boundary layer, shear layer, vortex and wake are the five physical interacting parameters. Based on interaction mechanisms, the whole region of $\alpha$ and $T / D$, can be classified into seven regimes as illustrated in Fig. 3.

\subsection{No interaction regime}

Here the cylinder acts independently - like a single cylinder - and is not interfered by the other (Fig. 3(b)). This happens for $|\alpha| \approx 45^{\circ} \sim 180^{\circ}, T / D>1.3-5$ depending on $\alpha$. The values of drag, lift and Strouhal number are the same as those of a single isolated cylinder.

\subsection{Boundary-layer (BL) and cylinder interaction regime}

Interaction between boundary layer and cylinder is generally strong and afoot when the two cylinders are very close $T / D<0.3-0.6$ depending on $\alpha$. Interacting with the other cylinder, the boundary layer of a cylinder may form separation bubbles, delay to separate, reattach, bifurcate, swerve, switch, etc (Fig. 3(c)). Therefore the interaction not only intensifies $C_{L}$ but also causes a bistable nature of flow (Alam and Meyer 2011). Figure 4 displays how strong the dependence of $C_{L}$ on $\alpha$ is. As $\alpha$ increases from $0^{\circ}$ to $180^{\circ}, C_{L}$ is negative for $0^{\circ}<\alpha<45^{\circ}$ with a minimum at $\alpha=10^{\circ}$, positive for $45^{\circ}<\alpha<135^{\circ}-155^{\circ}$ depending on $T / D$ and then again negative for $135^{\circ}-155^{\circ}<\alpha<180^{\circ}$, with a global maximum and minimum at $\alpha=135^{\circ}$ and $\alpha=155^{\circ}$ for $T / D=0.1$ and 0.3 , respectively. The adhered flow structures explain why $C_{L}$ follows such a trend. For $0^{\circ}<\alpha<45^{\circ}$, the boundary layer from the upper side of cylinder $A$ reattaches on the upper side of cylinder $B$ and bifurcates (Fig. 4(b)). One part goes to the lower side and 
travels for a longer peripheral length before its separation, producing a net anticlockwise circulation, thus corresponding to the negative $C_{L}$. Furthermore, the reattachment point corresponds to the stagnation point; hence a relatively positive pressure occurs on the upper side which also contributes to the negative $C_{L}$. With increase in $\alpha$ up to $90^{\circ}$ the lateral gap between the cylinders increases, hence the stagnation point moves to the lower side (Alam et al. 2003a; Alam and Zhou 2007), producing positive $C_{L}$ (Fig. 4(c)). A further increase in $\alpha$ corresponds to a decrease in the lateral gap. Therefore, pushed back by cylinder $A$, the lower boundary layer of cylinder $B$ reattaches on the rear surface of the same cylinder, (Fig. 4(d)), forming a separation bubble. A large negative pressure is in general accompanied by a separation bubble. Hence the net lift is downward, negative.

\subsection{Shear-layer/wake and cylinder interaction regime}

The interaction occurs when two cylinders are nearly in tandem, $|\alpha| \approx 0^{\circ} \sim 20^{\circ}, 0.3<T / D<2.3-3$. The shear layer(s) from one cylinder directly interact(s) on the other cylinder surface by reattaching, impinging, forming a separation bubble, etc. Naturally, one of the cylinders is completely or partially submerged in the wake of the other, hence it can also be termed wake and cylinder interaction (Figs. 3d-f). $C_{L f}$ is small at this regime except at a small island $\left(T / D=1 \sim 2, \alpha \approx 0^{\circ}\right)$ where $C_{L f}$ is slightly higher. While the small $C_{L f}$ corresponds to a steady reattachment of the upstream-cylinder shear layer(s) on the downstream cylinder (Figs. 3d, f), the other is ascribed to an alternating reattachment (Fig. 3(e)). Thus a strong interaction between shear-layer and cylinder takes place. Being completely or partially submerged in the wake of the other, the cylinder acting as a stabiliser suppresses the flow unsteadiness between the cylinders (Figs. 3d, f). This regime corresponds to high $C_{L}$ because the high velocity slice of the upstream shear layer goes beneath the downstream cylinder (Fig. 3(d)).

\subsection{Shear layer (SL) and shear layer (SL) interaction regime}

This interaction occurs at slightly higher $\alpha$ than shear-layer/wake and cylinder interaction. Here the shear layer(s) of one cylinder directly interact(s) with that of the other (Fig. 3(g)). The interaction causes intermittent interlock-in of the shear layers, hence generating vortices at more than one frequency 
(Alam and Sakamoto 2005), and reducing both fluctuating and mean forces on the cylinders. The two shear layers interact with themselves and the outer-shear layers.

\subsection{Vortex and cylinder interaction regime}

This interaction takes place at $\alpha \approx-20^{\circ} \sim 20^{\circ}, T / D>2.1-3$. The critical spacing is $T / D=3$ at $\alpha$ $\approx 0^{\circ}$, decreasing to 2 as $\alpha$ increases to $20^{\circ}$. When $T / D$ is greater than the critical spacing of two nearly in-line cylinders, the shear layers of the upstream cylinder cannot reach the downstream cylinder, hence they roll between the cylinders, forming alternate vortices (Alam et al, 2003b). The alternate vortices from the upstream cylinder subsequently strike on the downstream cylinder and embrace the side surface during passing on the cylinder (Fig. 3(h)). This interaction is generally very strong, and it intensifies fluctuating lift and drag significantly.

\subsection{Vortex and shear-layer (SL) interaction regime}

The interaction happens at $|\alpha| \approx 20^{\circ} \sim 35^{\circ}, T / D>2.1$. For this large $\alpha$, the downstream cylinder becomes offset from the inner row of vortices from the upstream cylinder. The result is that, the vortices cannot interact with the downstream cylinder, but they can interact with the inner-shear layer of the downstream cylinder. Interacting with the shear layer while it is growing, the vortices force the shear layer to form a synchronized coupled vortex (Fig. 3(i)). This interaction renders a very high fluctuating lift, as alternate interaction between vortex and shear layer intervenes.

\subsection{Vortex and vortex interaction regime}

For a further increase in $\alpha$, the transverse distance between the cylinders becomes large, and each cylinder forms a separate wake immediately behind them (Fig. 3(j-1)). The vortices on the two inner rows interact with each other and combine the two wakes into a wider one, which results in a slightly higher fluctuating lift and drag.

\section{Flow-induced instability}

How the interactions affect flow-induced instability of the twin cylinders - compared to a single isolated (non-interfering) cylinder - is of great interest to researchers in science and engineering. This 
section includes an overview of flow-induced vibration results for two elastically mounted cylinders. The detailed results of cylinder responses at different interaction regimes are presented in Fig. 5. While the vertical axis of the response curves represents the vibration amplitude $a$ normalized by $D$, the horizontal axis is $U_{r}$. The response curves were incorporated from Bokaian and Geoola (1984a, b), Kim et al. (2009) and Alam and Kim (2009), yet mostly from the latter two. The dashed line in the response graphs stands for single isolated cylinder response, insinuating VE at $U_{r} \approx 5.4(\approx 1 / \mathrm{St}=1 / 0.186)$. While both cylinders experience divergent galloping vibration for $U_{r}>10$ at $0<\alpha<25^{\circ}$ (Fig. 5(a, d)) in the boundary layer and cylinder interaction regime, they experience VE between $U_{r}=7$ to 10 for $25^{\circ}<\alpha<155^{\circ}$ (Fig. 5(b, c)). For the latter case, the downstream cylinder vibration amplitude is larger than the upstream one. Divergent violent vibrations of both cylinders are generated in the regime of shear-layer/wake and cylinder interaction (Fig. 5(e, f, n, o)). VE and galloping are combined at smaller T/D (Fig. 5(e, o)) and separated for larger $T / D$ (Fig. 5(f)). High amplitude VE is afoot in the regimes of vortex and cylinder interaction (Fig. 5(g)) and vortex and shear-layer interaction (Fig. 5(h)), where $C_{L f}$ on stationary cylinders is high (Fig. 2(b)). In the SL and SL interaction regime, VE occurs at two regimes of $U_{r}$ (Fig. 5(i, 1)). Each cylinder sheds vortices at two frequencies (Alam and Sakamoto 2005), hence experiences two VE. In the vortex and vortex interaction regime, VE intervenes at a high $U_{r}$ for the downstream cylinder (Fig. 5(j)) and at a low $U_{r}$ for the upstream cylinder (Fig. $5(\mathrm{k})$ ). This is due to the fact that the downstream and upstream cylinders generally shed vortices at a low and at a high frequency, respectively. The no interaction regime corresponds to VE at the same $U_{r}$ as that of a single cylinder (Fig. 5(m)).

It is worth mentioning that a larger $C_{L f}$ (Fig. 2(b)) corresponds to larger amplitude VE (Fig. 5(c, g, h)). The most striking feature is that divergent galloping vibration is generated at shear layer/wake and cylinder interaction (Fig. 5(e, f, n, o) ) and at boundary layer and cylinder interaction (Fig. 5(a, d)) regimes where there is a large variation in $C_{L}$ in the cross-flow direction (Fig. 2(a)). Based on galloping theories it is an acknowledged fact that galloping is not generated on an axis-symmetric body, e.g. a circular cylinder. Hence the question arises, why do two circular cylinders in close proximity experience galloping? In the 
regimes of boundary layer and cylinder interaction as well as shear -layer/wake and cylinder interaction, the two cylinders are connected by boundary layer or shear layer, and the combined shape of the two cylinders is not longer axis symmetric, hence the two cylinders may be prone to generating galloping vibrations. Furthermore, due to having non-uniform velocity between the cylinders, the downstream cylinder is again not axis symmetric with respect to local approaching flow. In other words, the galloping generation for two circular cylinders at close proximity is not violating the galloping theories. Details of the instability mechanism are discussed in the next section with reference to the lift force and interaction mechanisms.

\section{Mechanism of Instability}

Figure 6 shows $C_{L}$ variation with change in $y / D$ at $x / D=2 . C_{L}$ is maximum and minimum when $y / D=-0.4$ and 0.4 , respectively. These two $y / D$ values correspond to the locations of positive and negative peaks in the $C_{L}$ contour map (Fig. 2(a)). The figure suggests that when the cylinder position is below the center line $(y / D=0), C_{L}$ is in an upward direction; and when the cylinder position is above the center line, $C_{L}$ is in a downward direction. Now it is possible to get $\partial C_{L} / \partial(y / D)$. Figure 7 shows how $\partial C_{L} / \partial(y / D)$ varies with $y / D$. It is clear that the system is stable for $y / D=-0.4 \sim 0.4$ and unstable for $|y / D|>0.4$, following quasi-steady assumption. In the former region, our intuition is confirmed: in the case of the tandem cylinder $(y / D=0)$, when the downstream cylinder is displaced in the transverse direction away from $y / D=0$ line, there is a restoring lift force that is acting to return the cylinder to its original position (see Fig. 6). Hence quasi-steady arguments, as used in galloping theory, suggest stability of the downstream cylinder rather than instability. Galloping type response however occurs at $y / D=-0.4 \sim 0.4$ (Fig. 5). Why and how? At $y / D=0, C_{L}=0$ (Fig. 6), there is no force to displace the cylinder in a transverse direction. But for $y / D \neq 0, C_{L} \neq 0$, i.e., a force exists to displace the cylinder from its neutral position. Cylinder motion is thus generated, though displacement may be very small. It does not matter in which direction the displacement occurs. The question that now arises is how an initial displacement for $y / D=0$ occurs. Indeed, $y / D=0$ is the critical geometry between staggered configurations of $y / D=0+$ and $y / D=0-$. 
For $y / D=0+$, only the upper shear layer of the upstream cylinder reattaches onto the upper surface of the downstream cylinder; for $y / D=0-$, only the lower shear layer of the upstream cylinder reattaches onto the lower surface of the downstream cylinder. Hence, for $y / D=0$, the upper and lower shear layers of the upstream cylinder reattach alternately onto the upper and lower surfaces of the downstream cylinder, respectively, especially for $T / D$ smaller than critical spacing. This alternating reattachment generates fluctuating forces to displace the cylinder. When the cylinder is slightly displaced (Fig. 8(a)), the reattached shear layer is in a hesitating position, critically hovering to go on the upper side or the lower side. Instability is thus generated. For a cylinder spacing larger than critical, the oncoming vortex also has two options of where to go, on the upper side and lower side (Fig. 8(b)). This hesitation is responsible for generating the instability.

\section{Conclusions}

Time-mean lift, fluctuating lift, flow structures and the flow-induced responses of two circular cylinders are hooked up with mechanisms of interaction between the cylinders for all possible arrangements. The current investigation has led to the conclusions below.

Fluid dynamics around two cylinders is classified into seven based on how the two cylinders interact with each other. The seven occur at seven different interaction regimes, namely no interaction regime; boundary layer and cylinder interaction regime; SL/wake and cylinder interaction regime; SL and SL interaction regime; vortex and cylinder interaction regime; vortex and SL interaction regime; and vortex and vortex interaction regime. Each of them has different traits and is connected to a different flow-induced response. While forces and flow-induced responses are similar to those of a single isolated cylinder in the no interaction regime, lift force is intensified and galloping vibration is generated in the boundary layer and cylinder interaction regime. In the SL/wake and cylinder interaction regime, lift varies briskly with $\alpha$ or $y / D$, fluctuating lift is reduced and galloping vibration is generated, in addition to VE. Two VEs occur at two different reduced velocities in the SL and SL interaction regime. Both vortex and cylinder interaction and vortex and shear layer interaction causes extensively high fluctuating lift, 
generating relatively high amplitude VE. The VE-reduced velocity is slightly higher for the vortex and cylinder interaction than for the vortex and shear layer interaction. Vortex and vortex interaction results in a slightly higher fluctuating lift and generates VE only.

Though a single non-interfering circular cylinder does not experience galloping, two circular cylinders incur violent galloping vibration due to SL/wake and cylinder interaction as well as boundary layer and cylinder interaction. A stronger fluctuating lift corresponds to a larger vibration VE.

\section{References}

Alam, M.M., Kim, S., 2009. Free vibration of two identical circular cylinders in staggered arrangement. Fluid Dynamic Research 41, 035507, 17pp.

Alam, M.M., Meyer, J.P., 2011. Two interacting cylinders in cross flow. Physical Review E 84, 056304, pp.16.

Alam, M.M., Moriya, M., Sakamoto, H., 2003a. Aerodynamic characteristics of two side-by-side circular cylinders and application of wavelet analysis on the switching phenomenon. Journal of Fluids and Structures $18,325-346$.

Alam, M.M., Moriya, M., Takai, K., Sakamoto, H., 2003b. Fluctuating fluid forces acting on two circular cylinders in a tandem arrangement at a subcritical Reynolds number. Journal of Wind Engineering and Industrial Aerodynamics 91, 139-154.

Alam, M.M., Sakamoto, H., 2005. Investigation of Strouhal frequencies of two staggered bluff bodies and detection of multistable flow by wavelets. Journal of Fluids and Structures 20(3), 425-449.

Alam, M.M., Zhou, Y., 2007. Flow around two side-by-side closely spaced circular cylinders. Journal of Fluids and Structures 23(5), 799-805.

Alam, M.M., Zhou, Y., Wang, X.W., 2011. The wake of two side-by-side square cylinders. Journal of Fluid Mechanics 669, 432-471.

Alam, M.M., Sakamoto, H., Zhou, Y., 2005. Determination of flow configurations and fluid forces acting on two staggered circular cylinders of equal diameter in cross-flow. Journal of Fluids and Structures 
$21,363-394$.

Bokaian, A., Geoola, F., 1984a. Proximity-induced galloping of two interfering circular cylinders. Journal of Fluid Mechanics 146, 417-449.

Bokaian, A., Geoola, F., 1984b. Wake-induced galloping of two interfering circular cylinders. Journal of Fluid Mechanics 146, 383-415.

Brika, D., Laneville, A., 1997. Vortex-induced oscillations of two flexible circular cylinders coupled mechanically. Journal of Wind Engineering and Industrial Aerodynamics 69-71, 293-302.

Brika, D., Laneville, A., 1999. The flow interaction between a stationary cylinder and a downstream flexible cylinder. Journal of Fluids and Structures 13, 579-606.

Gu, Z., Sun, T., 1999. On interference between two circular cylinders in staggered arrangement at high subcritical Reynolds numbers. Journal of Wind Engineering and Industrial Aerodynamics 80, 287-309.

Hover, F.S., Triantafyllou, M.S., 2001. Galloping response of a cylinder with upstream wake interference. Journal of Fluids and Structures 15, 503-512.

Huera-Huarte, F.J. and Bearman P.W., 2011. Vortex and wake-induced vibrations of a tandem arrangement of two flexible circular cylinders with near wake interference. Journal of Fluids and Structures 27, 193-211

Kim, S., Alam, M. M., Sakamoto, H., Zhou, Y., 2009. Flow-induced vibrations of two circular cylinders in tandem arrangement, Part 1: characteristics of vibration. Journal of Wind Engineering and Industrial Aerodynamics 97, 304-311.

Kim, S., Alam, M.M., Sakamoto, H., Zhou, Y., 2009. Flow-induced vibrations of two circular cylinders in tandem arrangement, Part 2: suppression of vibration. Journal of Wind Engineering and Industrial Aerodynamics 97, 312-319.

Laneville, A., Brika, D., 1999. The fluid and mechanical coupling between two circular cylinders in tandem arrangement. Journal of Fluids and Structures 13, 967-986. 
Novak, M., 1969. Aeroelastic galloping of prismatic bodies. ASCE Journal of the Engineering Mechanics. Division 96, 115-142

Novak, M., 1972. Galloping oscillations of prismatic structures. ASCE Journal of the Engineering Mechanics. Division 98, 27-46

Parkinson, G., Smith J., 1964. The square cylinder as an aeroelastic non-linear oscillator Quarterly Journal of Mechanics and Applied Mathematics 17, 225-239.

Price, S.J., Paidoussis, M.P., 1984. The aerodynamic forces acting on groups of two and three circular cylinders when subject to a cross-flow. Journal of Wind Engineering and Industrial Aerodynamics $17,329-347$.

Simiu, E., Scanlan, R.H., 1996. Wind Effects on Structures. Fundamentals and Applictions to Design. Wiley-IEEE, New York.

Sumner, D., Price, S.J., Paidoussis, M.P., 2000. Flow-pattern identification for two staggered circular cylinders in cross-flow. Journal of Fluid Mechanics 411, 263-303.

Sumner, D., 2010. Two circular cylinders in cross-flow: A review. Journal of Fluids and Structures 26, 849-899.

Zdravkovich, M.M., 1987. The effects of interference between circular cylinders in cross flow. Journal of Fluids and Structures 1, 239-261.

Zdravkovich, M.M., Pridden, D.L., 1977. Interference between two circular cylinders; series of unexpected discontinuities. Journal of Industrial Aerodynamics 2, 255-270. 


\section{List of captions}

Fig. 1. Arrangement of cylinders and definitions of symbols.

Fig. 2. Contour maps of (a) time-mean lift coefficient $C_{L}$ and (b) fluctuating lift coefficient $C_{L f}$ of cylinder $B$. Points marked by ' $*$ ' denote values of coefficients of an isolated cylinder. Alam \& Meyer (2011).

Fig. 3. Interaction regimes in $T / D-\alpha$ plane. SL : shear layer, BL : boundary layer.

Fig. 4. (a) $C_{L}$ variation with $\alpha$ at $T / D=0.1$ (open circle) and 0.3 (open triangle); (b, c) and (d) flow structures corresponding to negative and positive $C_{L}$, respectively. $C_{L}$ corresponds to cylinder $B$.

Fig. 5. Flow-induced vibration response at different interaction regimes. Dashed line represents a single isolated cylinder response. The vertical and horizontal axes of the response graphs are the vibration amplitude ratio $a / D$ and reduced velocity $U_{r}\left(=U_{\infty} / f_{n} / D\right)$. The response curves are based on the results in Bokaian and Geoola (1984a, b), Kim et al. (2009), Borazjani and Sotiropoulos (2009), and Alam and Kim (2009) .

Fig. 6. $C_{L}$ variation with $y / D$ at $x / D=2.0$.

Fig. 7. $\partial C_{L} / \partial(y / D)$ variation with $y / D$ at $x / D=2.0$, corresponding to an extremely slow motion, i.e., $f_{n}=$ very low

Fig. 8. Instability generation for (a) $T / D<$ critical; (b) $T / D>$ critical. 


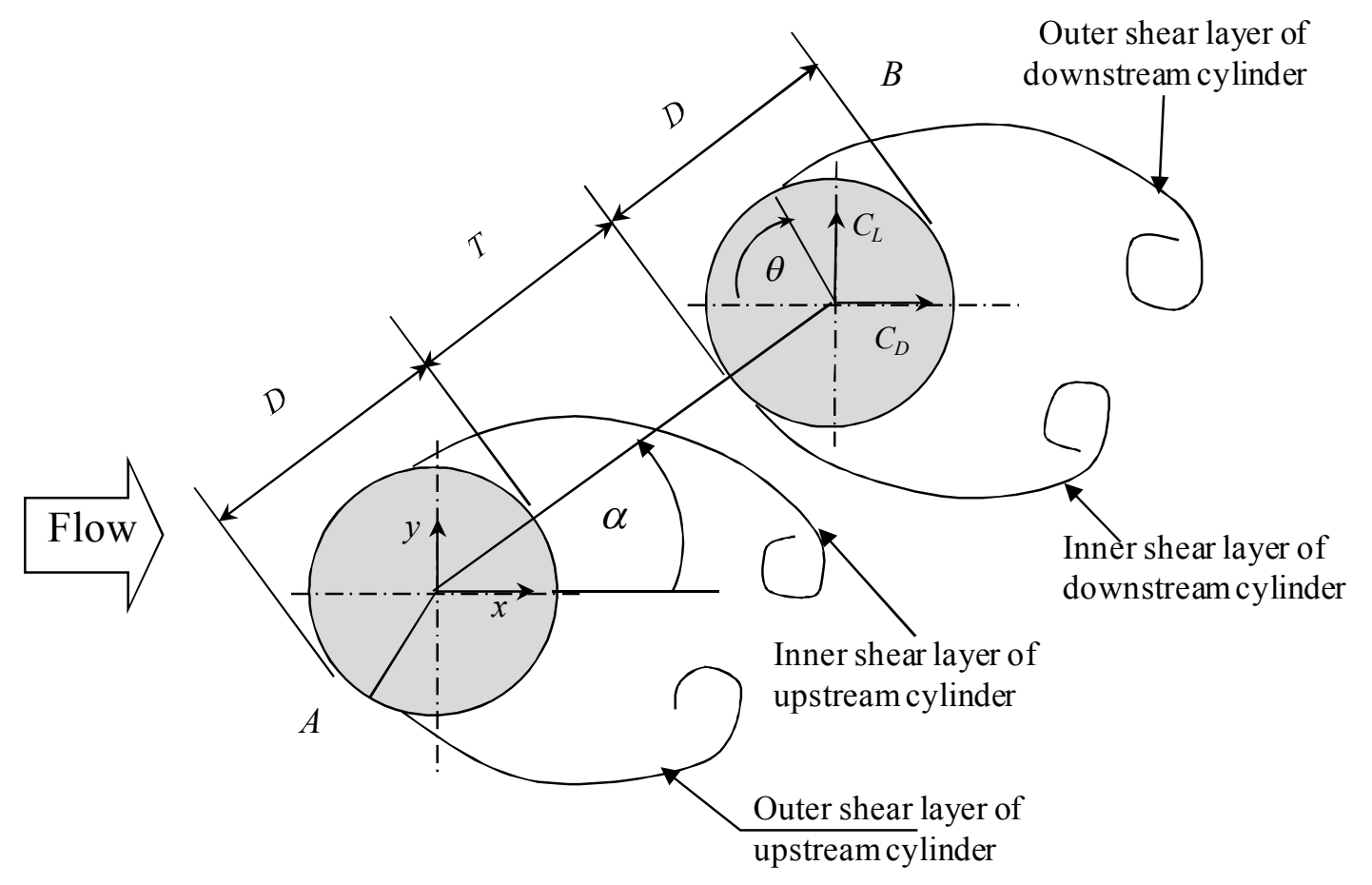

Fig. 1. Arrangement of cylinders and definitions of symbols. 

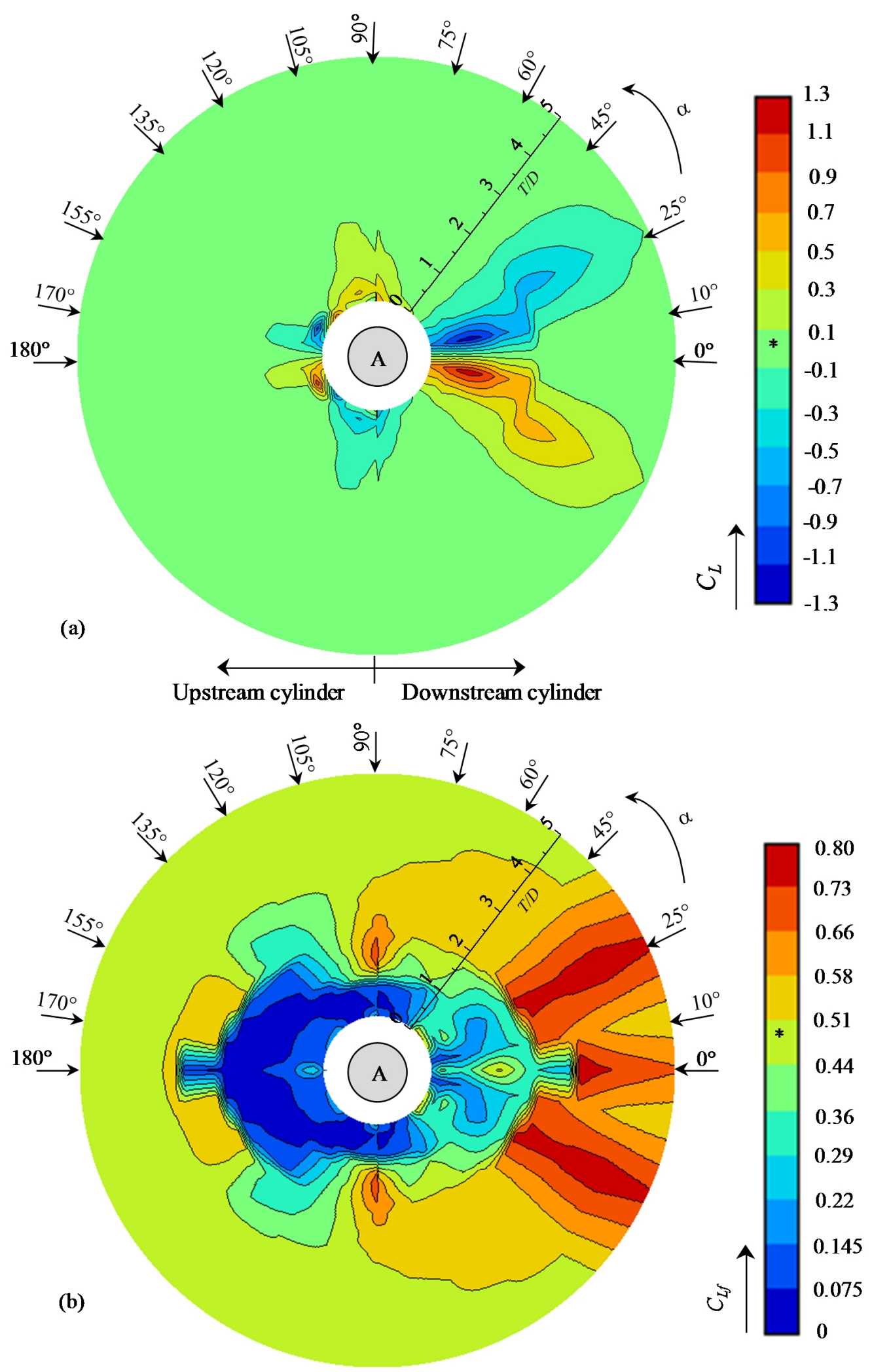

Fig. 2. Contour maps of (a) time-mean lift coefficient $C_{L}$ and (b) fluctuating lift coefficient $C_{I f}$ of cylinder $B$. Points marked by '*' denote values of coefficients of an isolated cylinder. Alam \& Meyer (2011). 


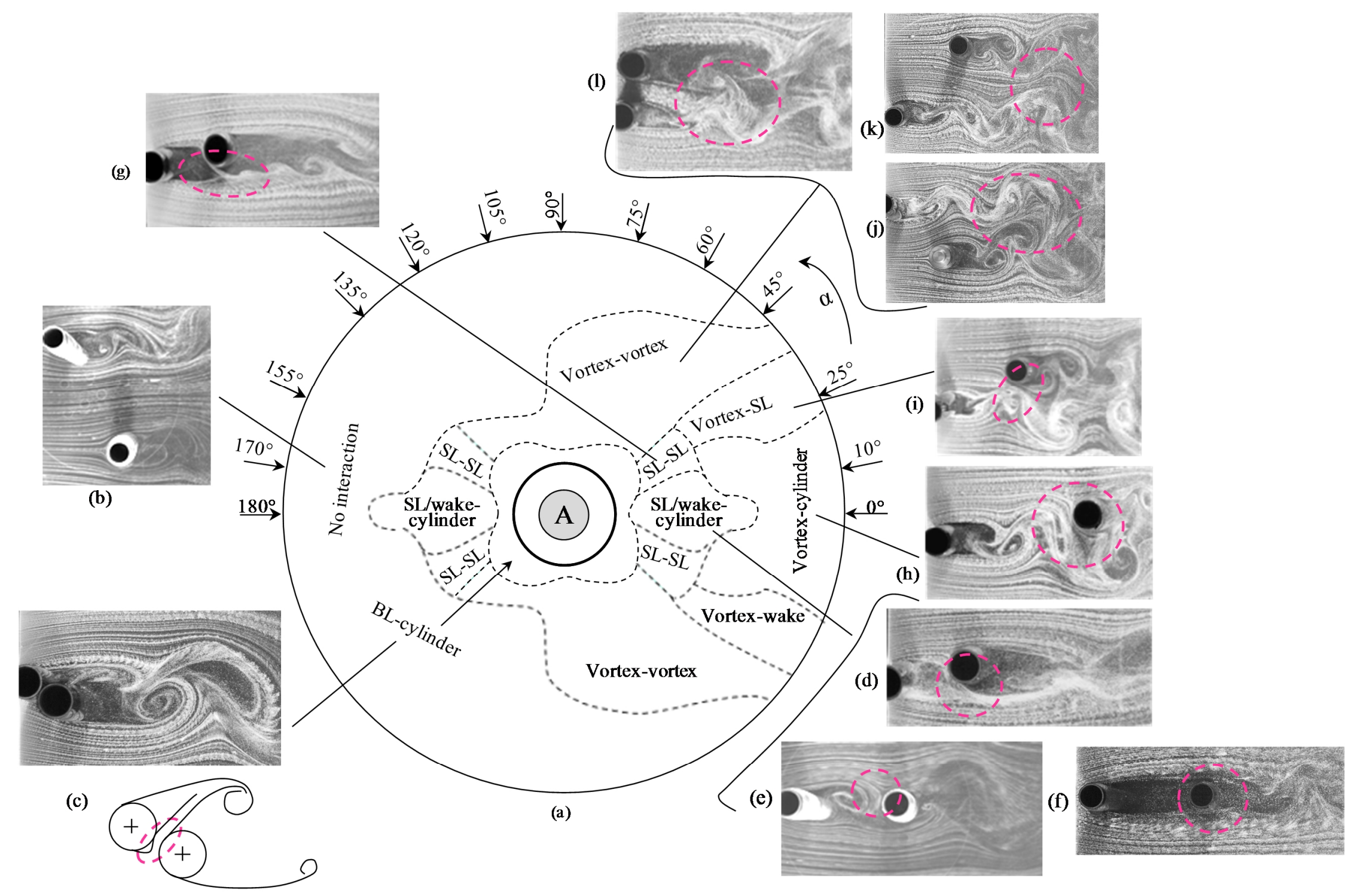

Fig. 3. Interaction regimes in $T / D$ - $\alpha$ plane. SL : shear layer, BL : boundary layer. 


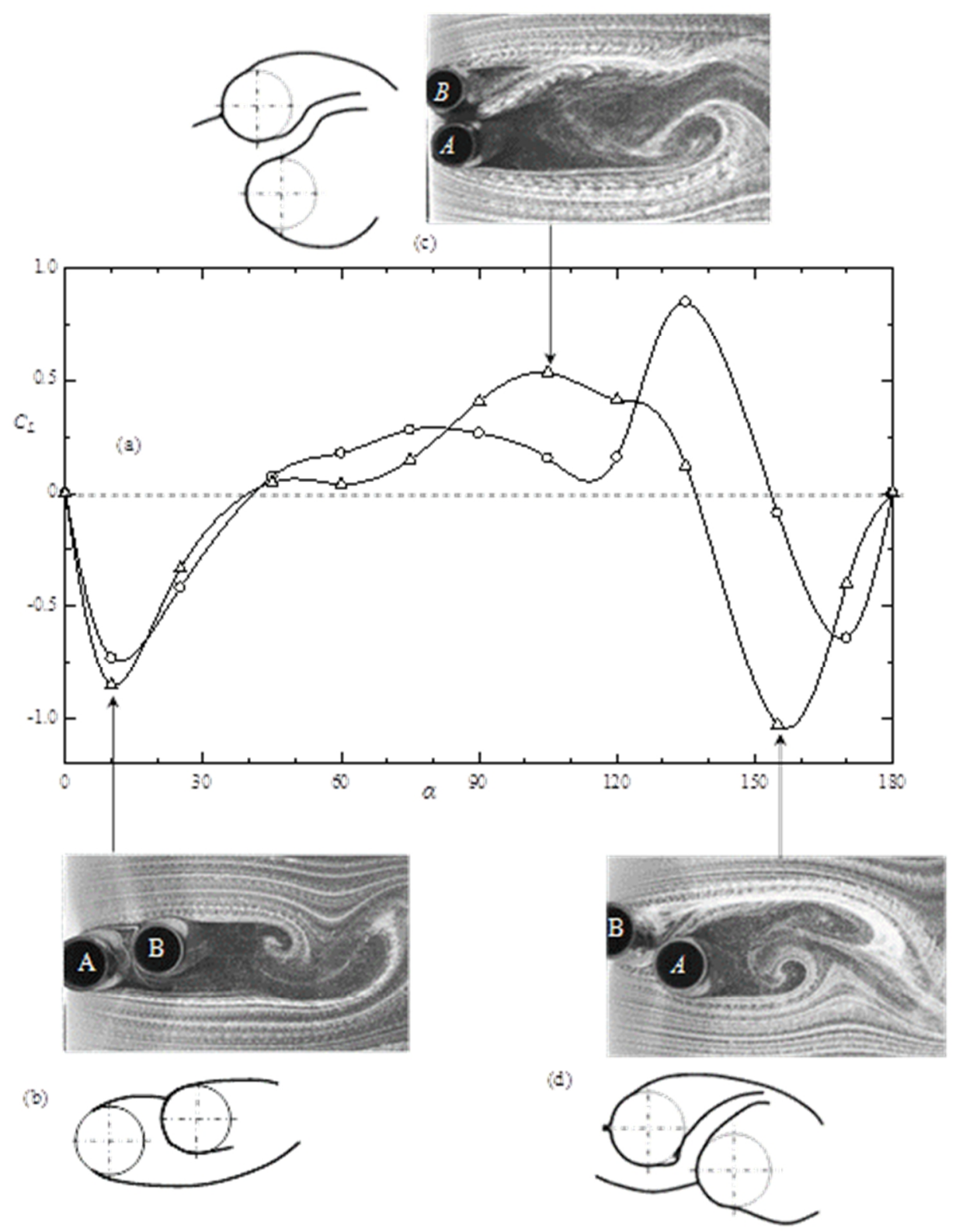

Fig. 4. (a) $C_{I}$ variation with $\alpha$ at $T D=0.1$ (open circle) and 0.3 (open triangle); (b, c) and (d) flow structures corresponding to negative and positive $C_{I}$, respectively. $C_{L}$ corresponds to cylinder $B$. 


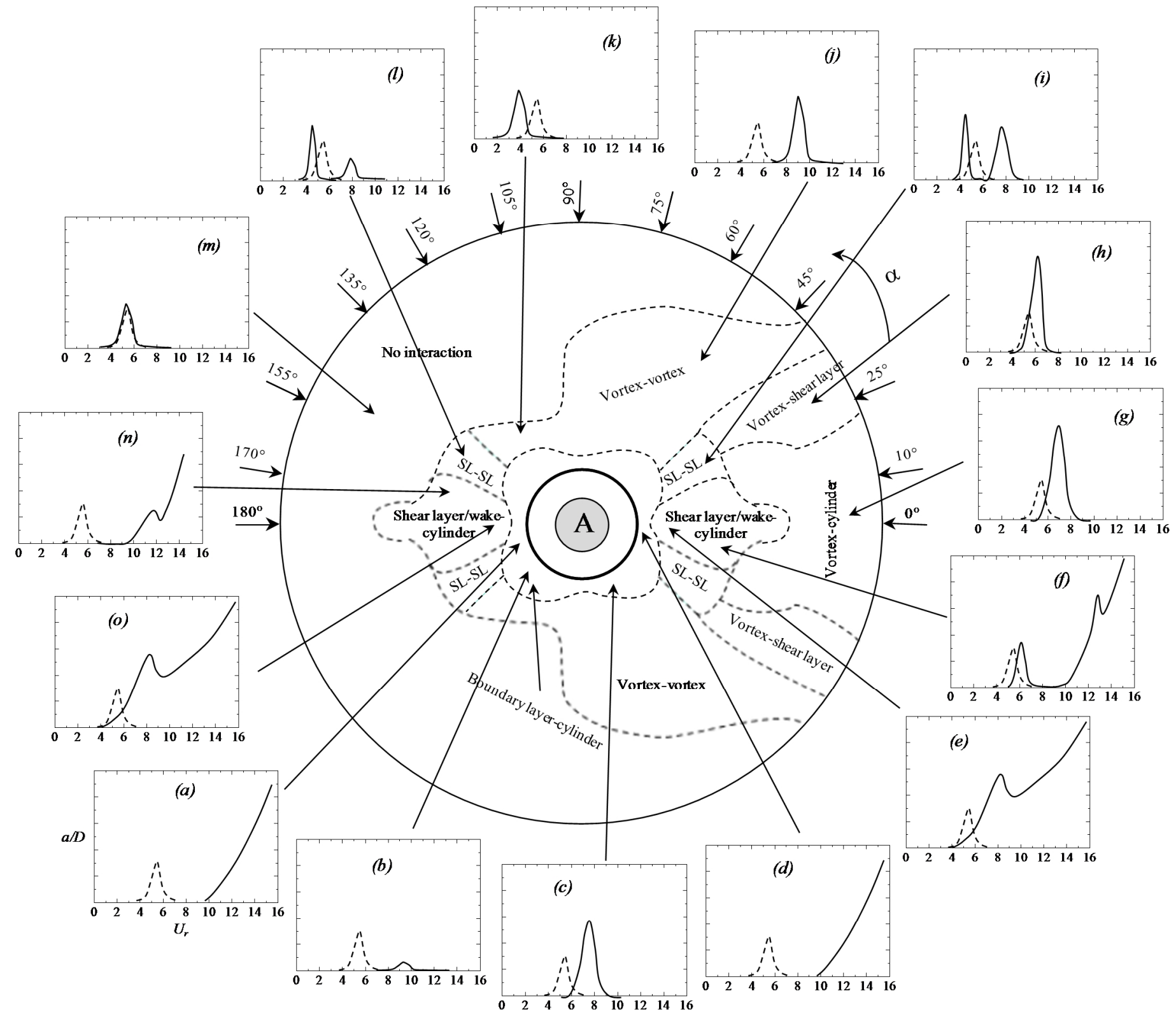

Fig. 5. Flow-induced vibration response at different interaction regimes. Dashed line represents a single isolated cylinder response. The vertical and horizontal axes of the response graphs are the vibration amplitude ratio $a / D$ and reduced velocity $U_{r}\left(=U_{\alpha} / f_{n} / D\right)$. The response curves are based on the results in Bokaian and Geoola (1984a, b), Kim et al. (2009), Borazjani and Sotiropoulos (2009), and Alam and Kim (2009). 

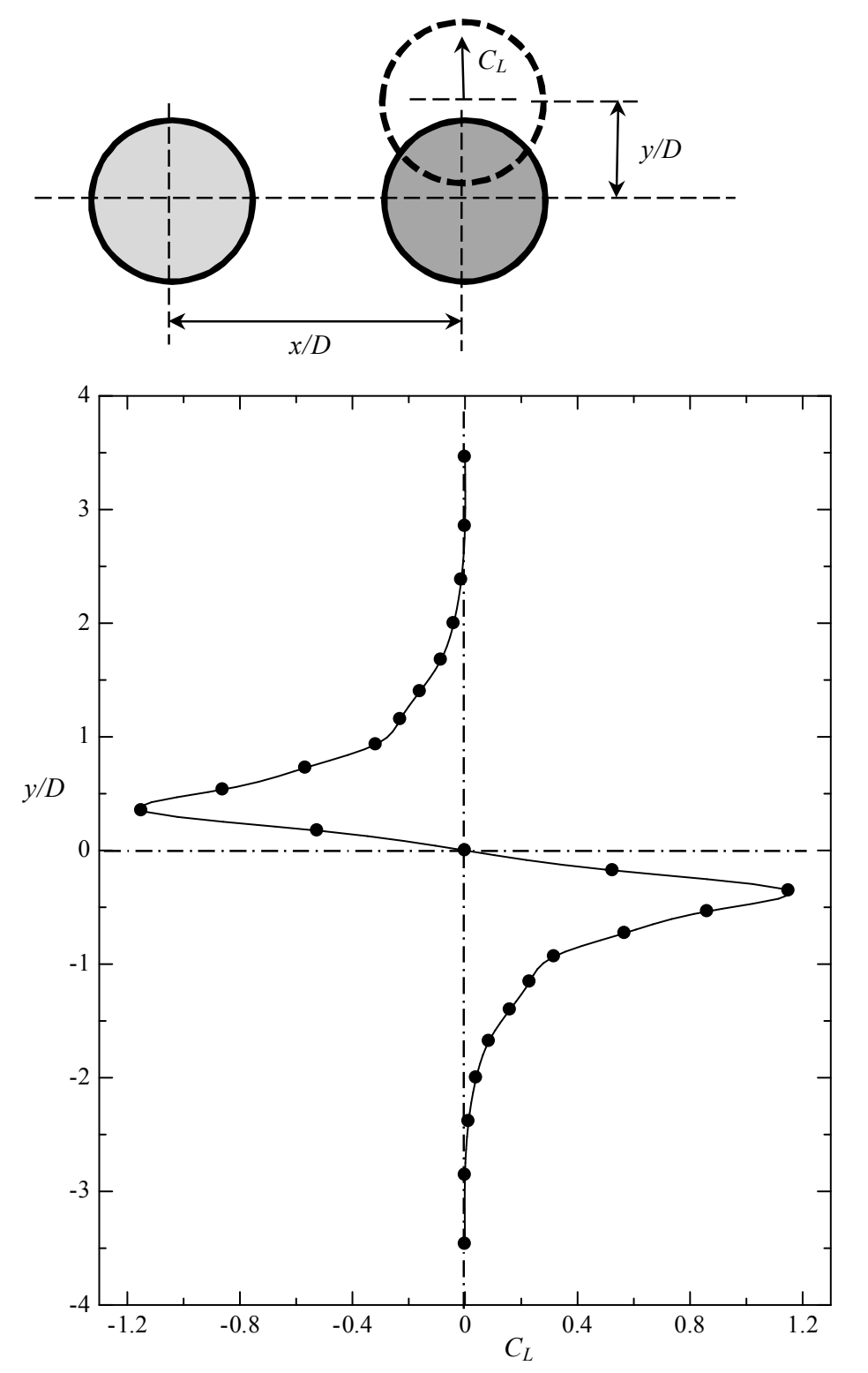

Fig. 6. $C_{L}$ variation with $y / D$ at $x / D=2.0$. 


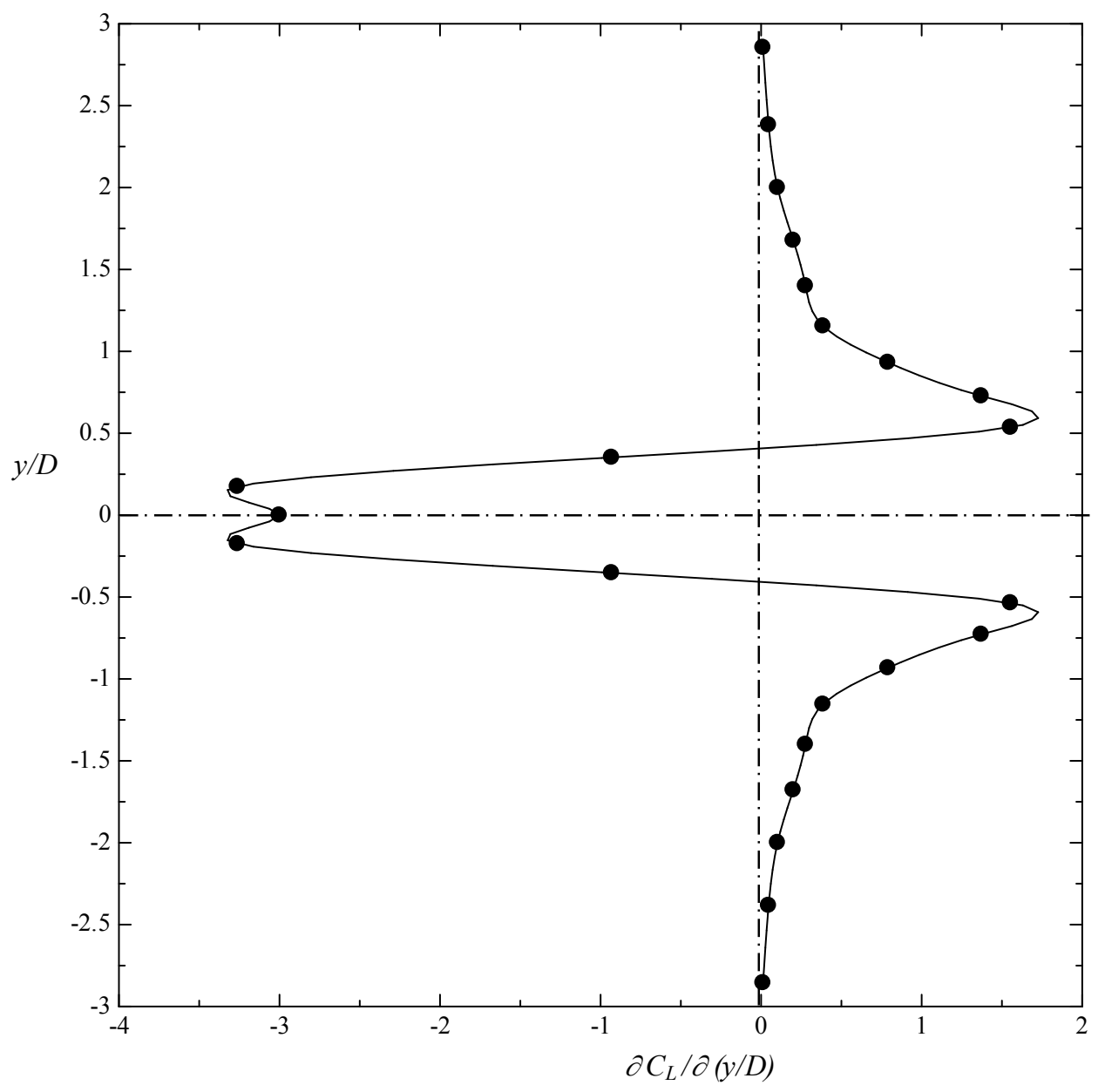

Fig. 7. $\partial C_{L} / \partial(y / D)$ variation with $y / D$ at $x / D=2.0$, corresponding to an extremely slow motion, i.e., $f_{n}=$ very low. 


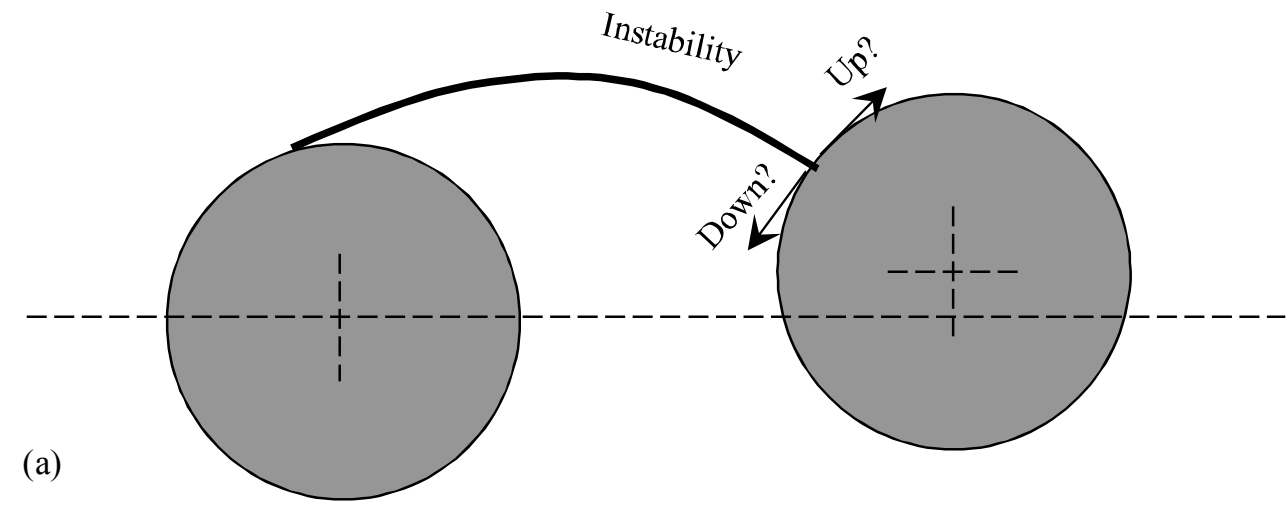

The shear layer is prone to switch from one side to the other
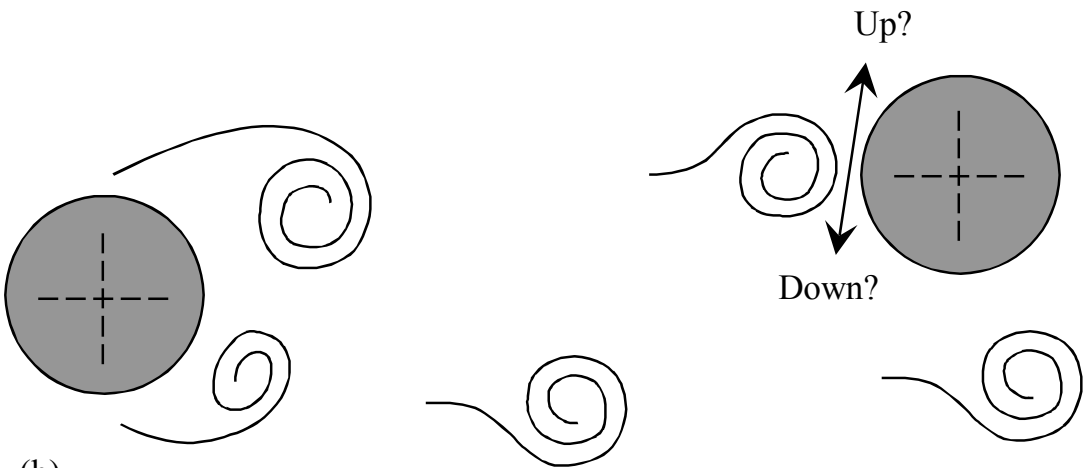

(b)

The vortex is prone to go to the upper and lower sides

Fig. 8. Instability generation for (a) $T / D<$ critical, (b) $T / D>$ critical. 